\title{
Biodiesel sensing using Silicon-on-Insulator technologies
}

\author{
Alvaro Casas Bedoya ${ }^{*}$, Meng Y. Ling, Joost Brouckaert, Nebiyu A. Yebo, Dries Van Thourhout, \\ Roel G. Baets \\ IMEC - Ghent University, Department of Information Technology, Sint-Pietersnieuwstraat 41, 9000 \\ Gent, Belgium
}

\begin{abstract}
By measuring the transmission of Biodiesel/Diesel mixtures in the near- and far-infrared wavelength ranges, it is possible to predict the blend level with a high accuracy. Conventional photospectrometers are typically large and expensive and have a performance that often exceeds the requirements for most applications. For automotive applications for example, what counts is size, robustness and most important cost. As a result the miniaturization of the spectrometer can be seen as an attractive implementation of a Biodiesel sensor.

Using Silicon-on-Insulator (SOI) this spectrometer miniaturization can be achieved. Due to the large refractive index contrast of the SOI material system, photonic devices can be made very compact. Moreover, they can be manufactured on high-quality SOI substrates using waferscale CMOS fabrication tools, making them cheap for the market.

In this paper, we show that it is possible to determine Biodiesel blend levels using an SOI spectrometer-on-a-chip. We demonstrate absorption measurements using spiral shaped waveguides and we also present the spectrometer design for on-chip Biodiesel blend level measurements.
\end{abstract}

Keywords: Silicon-on-Insulator, Biodiesel blends measurement.

\section{INTRODUCTION}

Biodiesel is a biofuel with methyl esters as a primary compound; these can be produced from different types of biomass, including animal fats, waste cooking oil and oilseed rape. Oilseed rape, in particular, is one of the principal sources of biofuel because it can be cultivated on a fairly wide range of agricultural land. Biofuel has become potentially important because it is a nontoxic, biodegradable and renewable source of energy that, in contrast to conventional Diesel fuel ${ }^{(1)}$, reduces the carbon dioxide $\left(\mathrm{CO}_{2}\right)$ emission in a combustion process. Therefore, the emission of greenhouse gases which are in big part responsible for the climate changes is also reduced.

Biodiesel can be used in Diesel engines as a pure fuel or in blends with Diesel fuel. To maintain optimum performance and meet emission regulations, it may be necessary to measure the composition of blended fuels and adjust engine parameters such as fuel injections timing during operation. ${ }^{(1)}$

Previous studies ${ }^{(2 ; 3 ; 4)}$ have showed that near- and far-infrared absorption spectroscopy are good tools to determine the Biodiesel/Diesel blend level. By measuring the absorbance of the mixture in these wavelength ranges, it is possible to predict the blend level with a high accuracy, as is shown in Fig. 1. This figure plots the transmission through a 1 centimeter cuvette filled with a mixture of Biodiesel/Diesel as a function of wavelength

*casas80@gmail.com; phone +32 488773794 


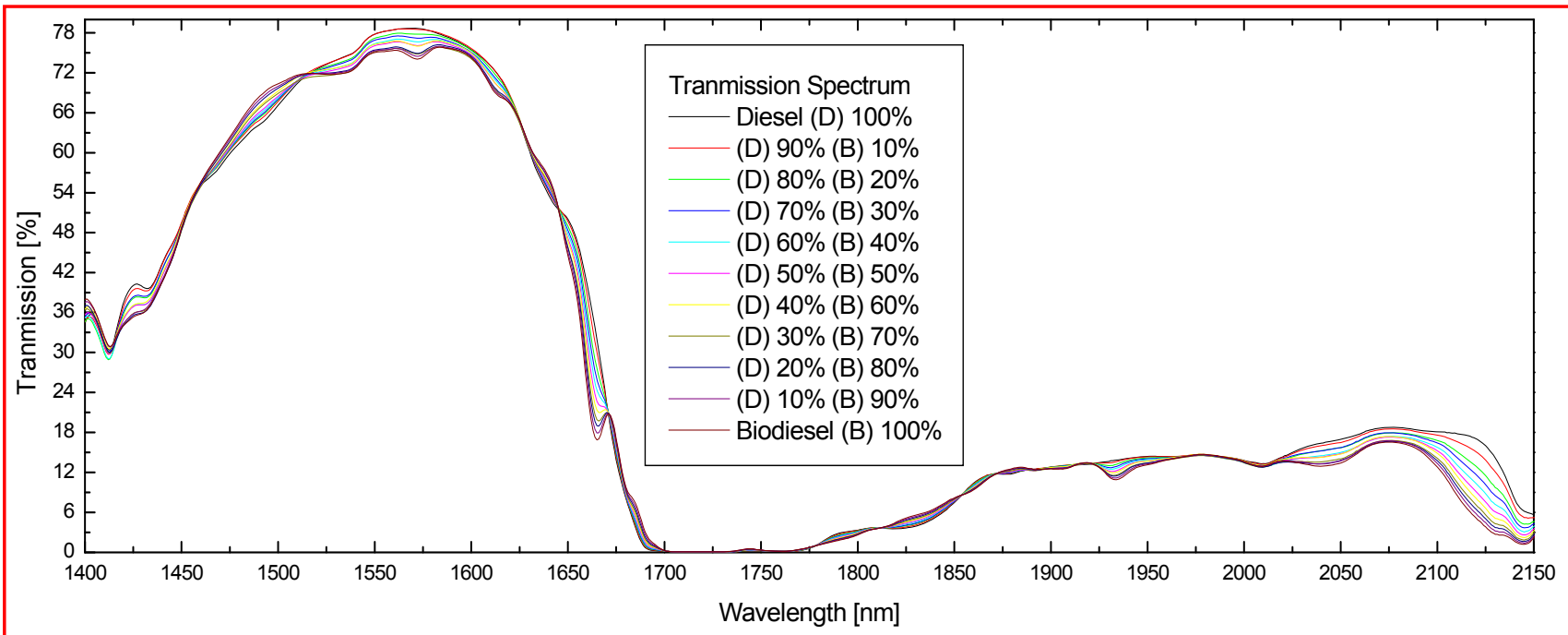

Fig. 1. Percentages of transmission for different blends of Biodiesel and conventional Diesel fuel with an optical path length of $1 \mathrm{~cm}^{(5)}$

Conventional photospectrometers are typically large and expensive and have a performance that often exceeds the requirements for most applications. For automotive applications for example, what counts is size, robustness and most important cost. As a result the miniaturization of the spectrometer can be seen as attractive Biodiesel sensor implementation, which could be able to adjust fuel injection timing during operation. A planar concave grating (PCG) is an interesting wavelength filter for the spectrometer, due to its possible small size, high resolution and large channel count. This devices has been realized in many material systems including silica-on-silicon, III-V's, large core siliconon-insulator (SOI) substrates and nanophotonic SOI substrates ${ }^{(6 ; 7 ; 8)}$. This last one is a promising material system for a wide range of applications, such as telecommunications and optical sensors. Due to the large omnidirectional index contrast of this material system, these devices can be made very compact and they can be manufactured on high-quality SOI substrates using CMOS fabrication tools, making these devices cheap for the market.

In this paper, we demonstrate the feasibility of implementing a Biodiesel blend level sensor on a SOI platform. We show that dedicated waveguide configurations can be used for absorption measurements and we discuss the design of an integrated grating based SOI spectrometer with an on-chip sensing area for this application. The paper is organized as follows: In Section II, we show and discuss the transmission spectra for different Biodiesel/Diesel mixtures in the nearinfrared wavelength range. In section III, we discuss the design of the chip. The chip consists of a waveguide sensing area and a spectrometer. We discuss the fuel measurement results in Section IV and we conclude in Section V.

\section{NEAR-INFRARED TRANSMISSION SPECTRUM OF BIODIESEL FUEL}

Using infrared spectroscopy the blend level of the Biodiesel/Diesel mixture can be analyzed. Different samples with different blend levels were prepared and characterized. The transmission spectra of the samples were recorded with a CARY 5000 UV-VIS-NIR photospectrometer by Varian. For these measurements, we used a plastic cuvette with an optical path length of $1 \mathrm{~cm}$ containing the Biodiesel/Diesel mixtures. Using the Cary WinUV software, we recorded transmission spectra, the recorded spectra were taken in the wavelength range of $1400 \mathrm{~nm}$ to $2400 \mathrm{~nm}$, with a resolution of $1 \mathrm{~nm}$, as is presented in Fig. 1.

The influence of the blend level on the transmission spectrum is clearly observed in Fig. 1. We are interested in the region of high and measurable differences in the blends absorptions. Therefore the most promising regions to determine the blends are around $2100 \mathrm{~nm}, 1930 \mathrm{~nm}$ and $1665 \mathrm{~nm}$. The transmission in the regions around $2100 \mathrm{~nm}$ and $1930 \mathrm{~nm}$ however is very low. The transmission in the $1665 \mathrm{~nm}$ region on the other hand is higher and from this point of view, this region is more interesting. In the $1665 \mathrm{~nm}$ region, a transmission difference of $20 \%$ is observed for the two fuel compounds, as is show below in Fig. 2. 


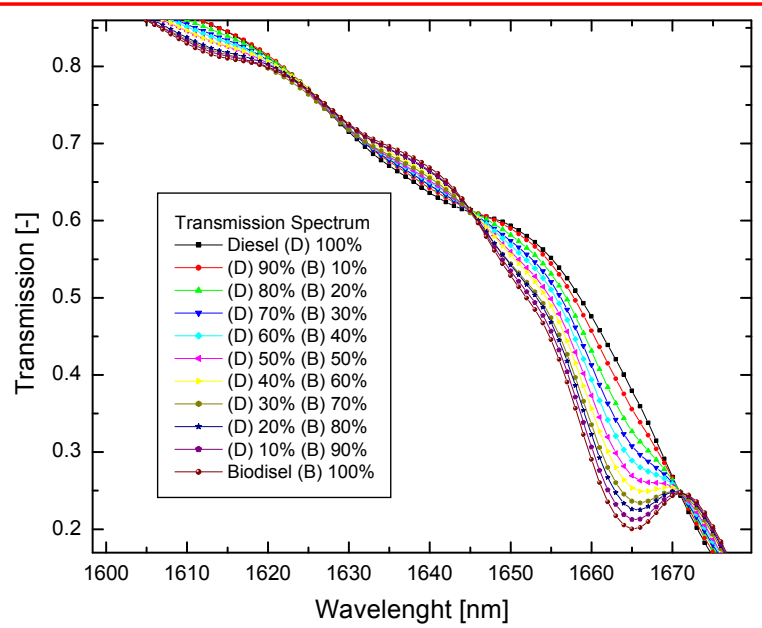

Fig. 2. Transmission spectrum in percentage of different blends of Biodiesel in conventional fuel

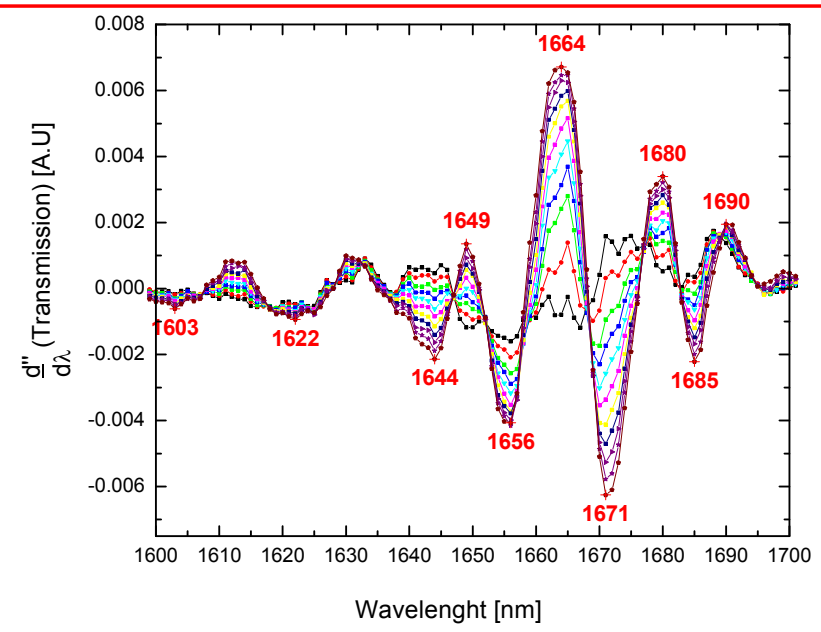

Fig. 3. Second derivative of the transmission spectrum around $1665 \mathrm{~nm}$

To define the number of wavelength channels of the PCG, a better spectral localization of these differences is needed. This localization can be done by taking the second derivative of the transmission spectrum in the $1665 \mathrm{~nm}$ region, (Fig. 3.). As can be seen, ten peaks at different wavelengths in the $1600-1700 \mathrm{~nm}$ region are identified. By designing a PCG with 14 output channels with a channel spacing of $7 \mathrm{~nm}$, each of these peaks can be reached.

\section{DESIGN OF THE SOI CIRCUIT}

The silicon-on-insulator material system we discuss in this work consist of a $220 \mathrm{~nm}$ thick Si waveguide layer on top of $2 \mu \mathrm{m}$ thick buried oxide layer on a Silicon substrate. By etching completely through the silicon waveguide layer, we obtain a very high refractive index contrast leading to a strong confinement. On the other hand the evanescent tail of the optical modes in the waveguides feel the fuel absorption processes. This interaction is proportional to the sensing length, and longer waveguides are necessary. These long lengths are achieved by curving the waveguide and creating a spiral shape waveguide. The interactions between the evanescent tail of the optical modes in the waveguides and the surrounding material are the key phenomenon in this sensing approach.

It is also possible to obtain the absorption information using structures which have a higher interaction with the surrounding material; this is the case for slotted waveguides. These waveguides are fabricated by etching a very narrow slot in the middle of a conventional waveguide and the intensity in the slotted area is very high ${ }^{(9)}$. The Biodiesel/Diesel absorption occurs in these waveguide configurations, and call them the sensing area in this work.

Previous research in our group reported a SOI-PCG ${ }^{(7)}$ fabricated in a complimentary metal-oxide-semiconductor line using deep-ultraviolet lithography, with four output channels separated by $20 \mathrm{~nm}$. The crosstalk was better than $-25 \mathrm{~dB}$ and the on-chip loss was drastically reduced down to $1.9 \mathrm{~dB}$ by replacing each facet by a second-order Bragg reflector. We adapted this design for the Biodiesel sensor application and we designed a 14 channel grating filter which meets the requirements mentioned in Section 2.

The SOI waveguide circuits were defined with $193 \mathrm{~nm}$ deep-UV lithography and transferred into the silicon using ICPRIE etching. For the definition of the grating demultiplexer, the photonic wire and slotted waveguides, we etched completely through the $220 \mathrm{~nm}$ thick Si layer. A more shallow etch $(70 \mathrm{~nm})$ was used for the definition of the $2 \mu \mathrm{m}$ wide entrance and exit waveguides of the PCG ${ }^{(8)}$. In the shallow etch step we also defined grating fiber couplers which allow for easy characterization ${ }^{(16)}$. The fiber couplers are one dimensional gratings which allow to couple nearly vertical incident light from a standard single mode fiber into the nanophotonic SOI waveguides. 


\subsection{Design of the 14 channel PCG with Distributed Bragg Reflector (DBR) type facets}

To measure the transmission at the different wavelengths of interest we have designed a 14 channel PCG and incorporated DBR grating facets. In our design, the DBRs have four periods of $670 \mathrm{~nm}$ in total, a trench width of $145 \mathrm{~nm}$ and an unetched part of $542.2 \mathrm{~nm}$. Fig. 5 is a top view photograph of a part of the grating

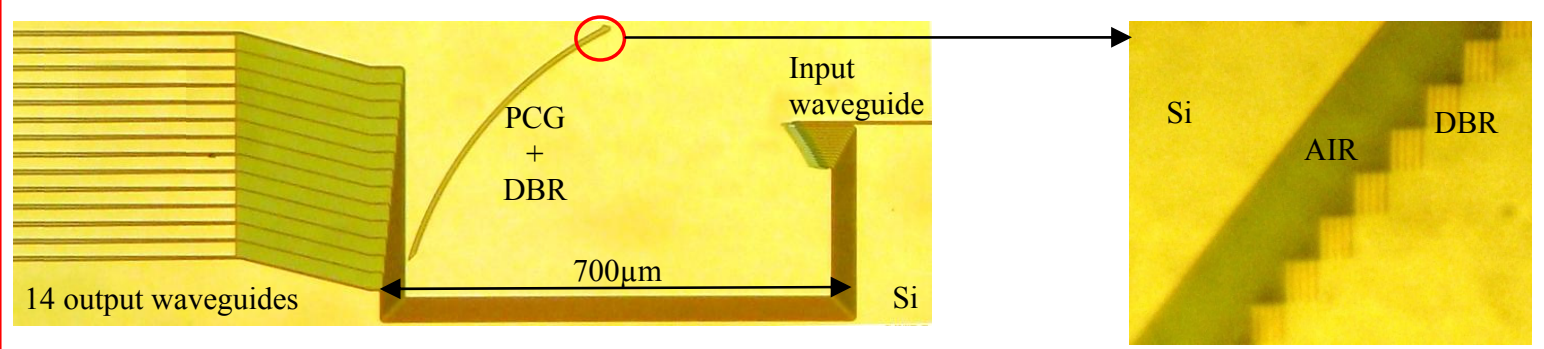

Fig. 4. Microscopic photograph of the SOI - PCG with 14 channels and

Fig. 5. Top view of DBR-type grating facets, with a width DBR

of $145 \mathrm{~nm}$. The unetched part is $542.2 \mathrm{~nm}$ wide

Fig. 6. Shows the transmission spectrum of a demultiplexer with one input waveguide and 14 output waveguides. The channel spacing is $7 \mathrm{~nm}$. The size of the diffraction grating, consisting of 91 facets totally, is about $440 \mu \mathrm{m}$.

The simulation of the PCG spectrum is done using scalar diffraction theory (Fig. 6.). For these simulations, we take into account a perfectly reflecting grating $(\mathrm{R}=1)$. This means these results give information on diffraction losses, crosstalk, channel spacing and loss variation over the total wavelength range. This design has a diffraction loss of about $0.5 \mathrm{~dB}$ for the central channel $(1650 \mathrm{~nm})$, and $30 \mathrm{~dB}$ crosstalk.

To calculate the total on-chip loss of the PCG, we also have to take into account the grating reflection loss. The reflection of the DBR facets is calculated using a 2-D fully vectorial solver based on eigenmode expansion CAvity Modelling FRamework $(\mathrm{CAMFR})^{(10)}$. The results from this simulation are presented in Fig. 7 as can be seen, the reflection at the central wavelength is $89 \%$. This corresponds with a grating reflection loss of $0.5 \mathrm{~dB}$.

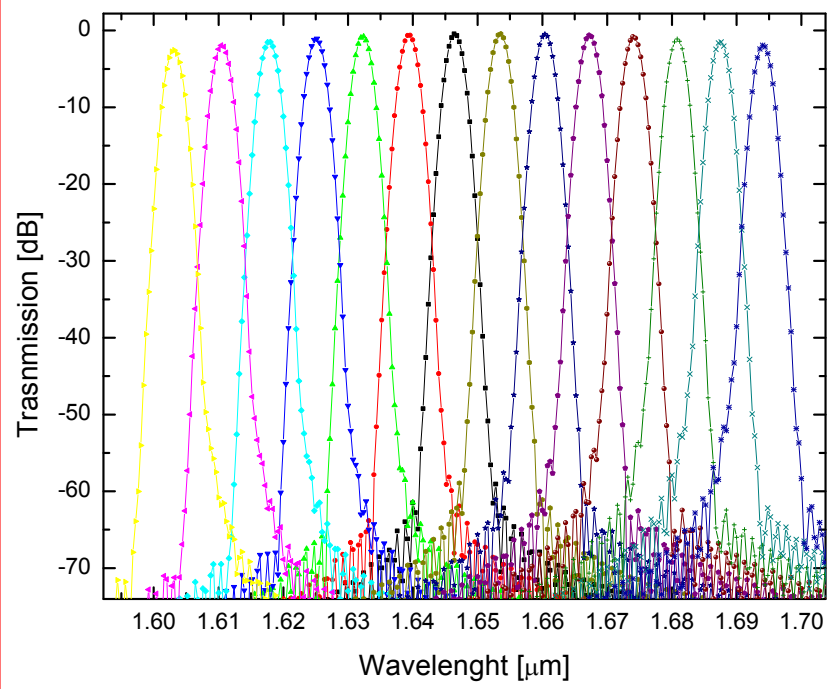

Fig. 6. Simulated transmission spectrum for 14 channels demultiplexer

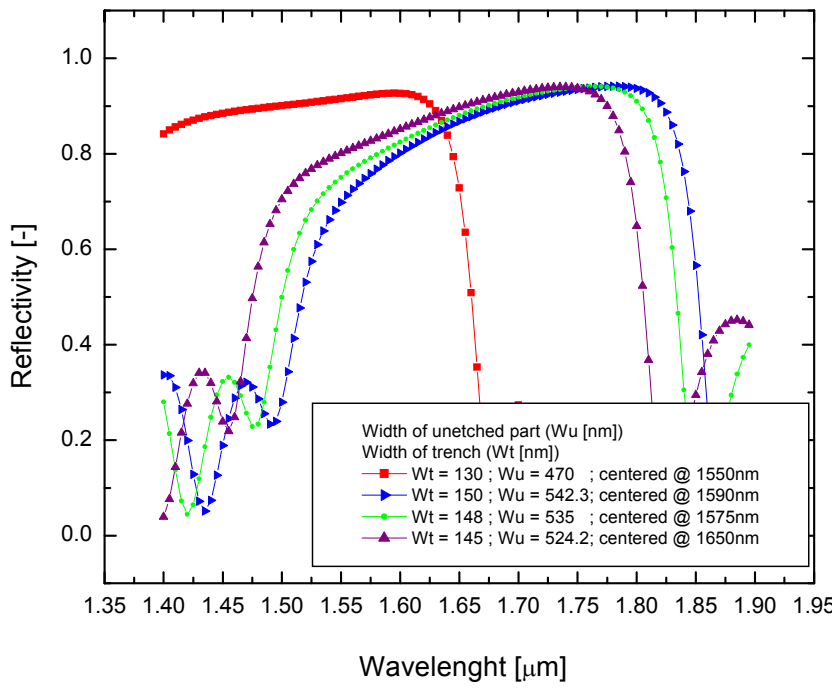

Fig. 7.Simulation results of the reflectivity of a four-period DBRtype facet. The period is $670 \mathrm{~nm}$ and the trench width varies from 130 to $150 \mathrm{~nm}$, the unetched part from 470 to 542.3 nm. Perfect vertical sidewalls are supposed 


\subsection{Design of the sensing area}

For first experiments, we measured the transmission of a broadband superluminescent LED through different waveguide configurations using a spectrum analyzer. For this purpose, we protected the chip area with SU-8, a photo-definable polymer and rectangular contact windows were opened on top of the sensing area as can be seen in figure 10 . In addition, a silicone box was also attached to the chip, keeping the fuel inside the open box and allowing to attach a flowcell on top of the chip (Fig 8). To be able to perform reproducible measurements, two single mode fibers were aligned and glued on top of the fiber couplers under a 10 degree angle from verticality using an UV-curable glue. This is shown in Fig. 9

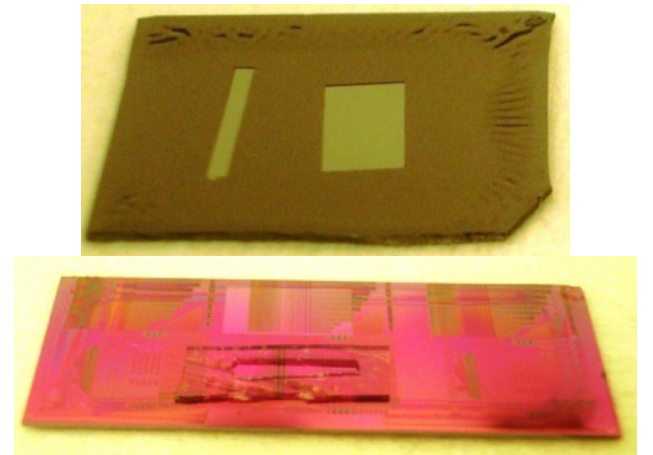

Fig. 8. Photograph of Si plus SU-8 and chip plus Silicone box

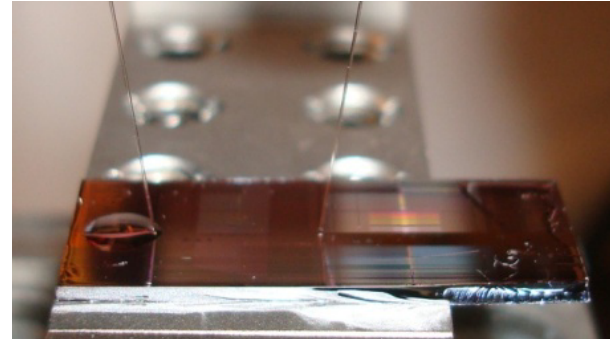

Fig. 9. Picture of the chip and the glued single mode fiber

The light interaction between the different waveguide configurations and the Biodiesel/Diesel blends can be described by the Beer-Lambert law. Taking into account the absorption of the material, and the interaction distance $x$, we create a model for the sensing area:

$$
P_{1}=P_{0} \cdot e^{-\Gamma \cdot \alpha \cdot x}
$$

Here $\Gamma$ is the confinement factor, $P_{0}$, and $P_{1}$, are the input and exit power and $\alpha$ is the absorption coefficient of the surrounding material. The confinement factor is a good measure for the amount of light that interacts with the surrounding fuel. This value is the fraction of the intensity in the fuel region over the total intensity.

In Fig. 10 the intensity profile of the propagating electric field is simulated for both, slotted and regular waveguides. These calculations were made using Fimmwave ${ }^{(11)}$, a fully vectorial mode solver. Here the intensity color map shows how the TE propagating mode is confined mostly in the core of the waveguide, however a large part of the electric field is also present in the cladding and in the free region; this last one, is where the evanescent tail of the optical mode feels the fuel absorption processes.

Fig. 11 shows simulations results of the confinement factors of both slotted and regular waveguides. The confinement factor is $70 \%$ and $20 \%$ respectively. 

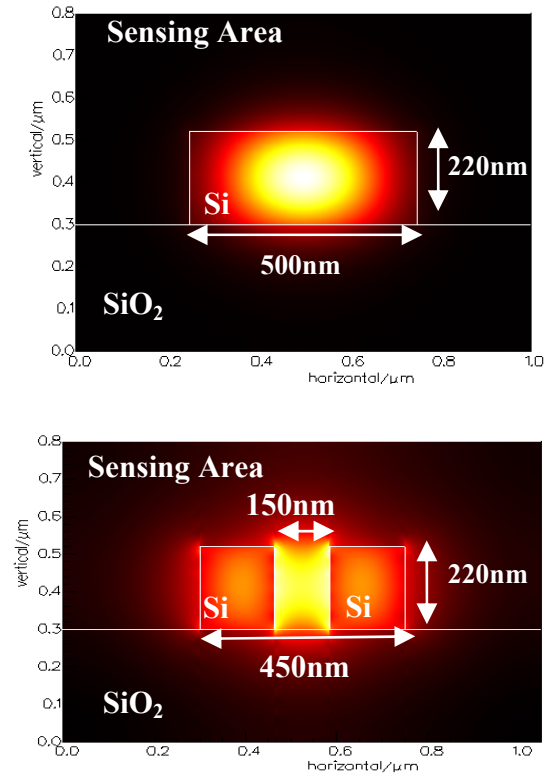

(b)

Fig. 10.Fimmwave simulation for the intensity profile of the fundamental TE-polarized mode in a Regular (a), and Slotted (b) waveguide. Using $\mathrm{n}_{\text {Biodiesel }}=1.455 ; \mathrm{n}_{\mathrm{Si}}=3.466 ; \mathrm{n}_{\mathrm{SiO} 2}=1.442$, refractive indices.

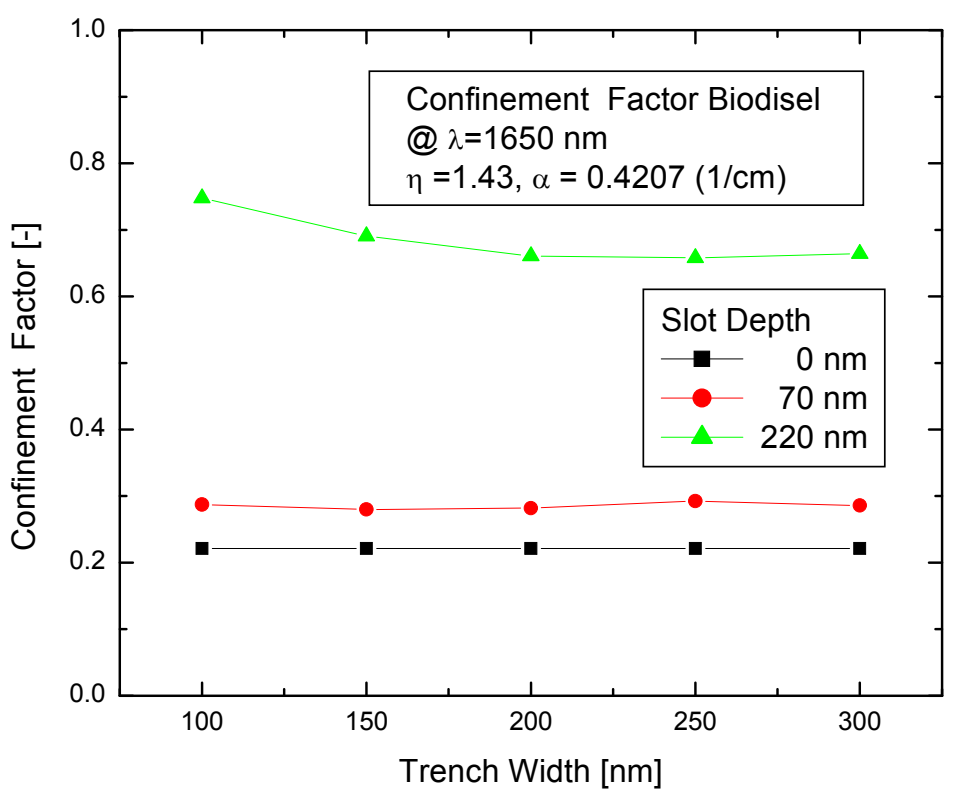

Fig. 11 Simulation results of the confinement factor of slotted and regular waveguides. The slot depth varies from 0 to $220 \mathrm{~nm}$ high. The width from 100 to $300 \mathrm{~nm}$

The differences in transmission are larger for longer waveguides, but the measurement are limited by different factors as: The power for the source, the coupling efficiencies, the losses on the chip and the power at the exit channels, moreover this last one should be large enough to be detected. Consequently the sensing area and its length is also limited by these values. These are already considered and the design is optimized. To find this length and based on simulations we have obtained that the fiber coupling losses are $(2 \times 4.877 \mathrm{~dB})$, for the central channel at the PCG the diffraction loss and the DBR loss are $0.5 \mathrm{~dB}$ in both cases, then total on-chip loss for the PCG is $1.0 \mathrm{~dB}$. The spiral waveguide have a propagation of $6.0 \frac{\mathrm{dB}}{\mathrm{cm}}$, the slotted has $14.0 \frac{\mathrm{dB}}{\mathrm{cm}}$ and finally a minimum detectable power of $100 \mathrm{nW}$ was imposed.

With all these values we obtained a maximum length available to incorporate in our device, which for regular waveguide is $1.10 \mathrm{~cm}$ and for slotted waveguides is $0.22 \mathrm{~cm}$.

The spiral photonics wire are already designed and incorporated to the PCG, as is explained in the Fig 12.

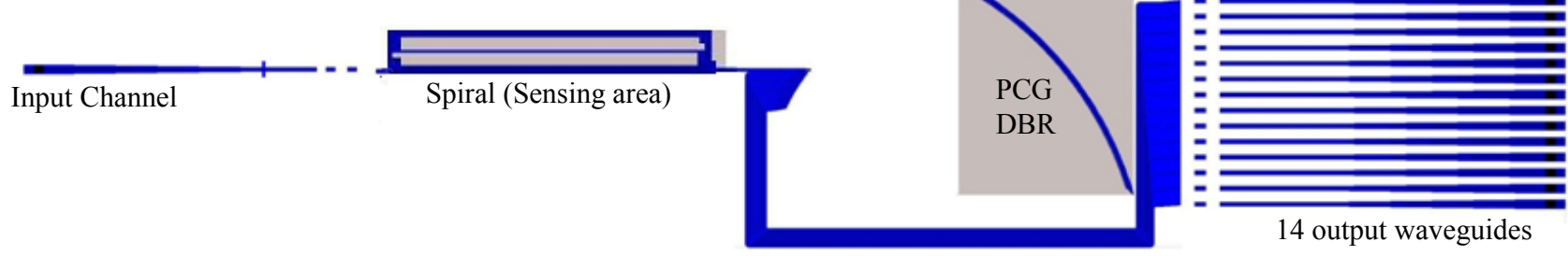

Fig. 12. Scheme of planar concave demultiplexer, plus a spiral photonics wire.

In the next chapter we report the measurements using the spiral shaped waveguides without the use of the PCG. 


\section{SENSING BIODIESEL USING SPIRAL SHAPED WAVEGUIDES}

In this chapter, we describe the measurement results of the fabricated samples. As mentioned earlier, the goal of these measurements is to investigate the possibility to determine Biodiesel blend levels by measuring the transmission through different waveguide configurations. For this experiment, we fabricated spiral shaped $500 \mathrm{~nm}$ wide photonic wire waveguides with different lengths. For these first experiments, we did not make use of a flow pump but fuel was added manually on top of the sensing box.

The transmission spectra from the chip were measured with an Optical Spectrum Analyser (OSA) with resolution setting of $0.1 \mathrm{~nm}$. A superluminescence broadband Light Emitting Diode (SLED) with a center wavelength at $1650 \mathrm{~nm}$ was used as the light source.

\subsection{Spiral waveguide characterization}

We measured the transmission spectrum of a set of four spirals with different lengths. (Fig. 13). The small oscillations are due to Fabry-Pérot effects caused by reflections at the fiber couplers ${ }^{(12)}$. The linear behavior observed in Fig. 14. was obtained by taking the maximum power transmitted for each spiral. With the linear regression we compare the losses and we calculated a propagation loss of $5.80 \pm 0.27 \underline{\mathrm{dB}}$ for the wires without Biodiesel on top.

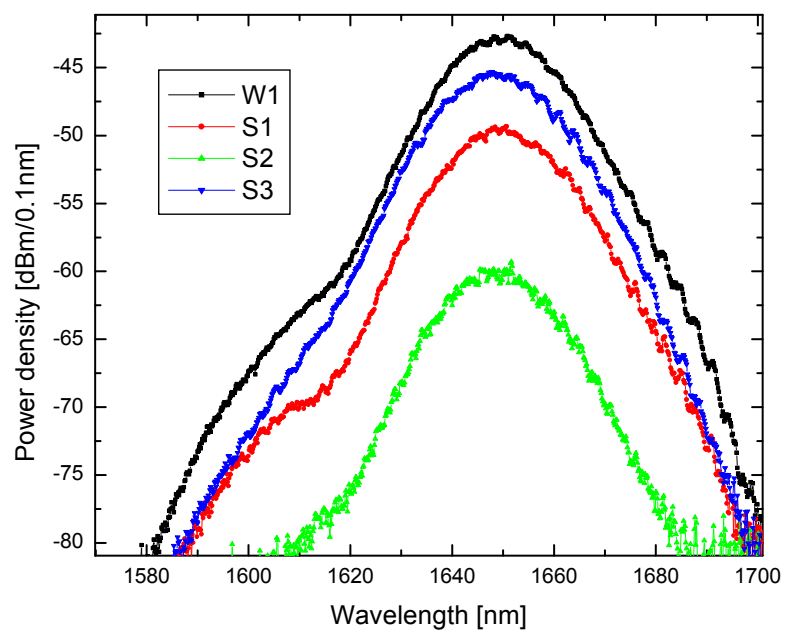

Fig. 13. Transmission spectra for spiral shaped photonic wire waveguides with different lengths ranging from $7 \mathrm{~mm}$ to 36 $\mathrm{mm}$.

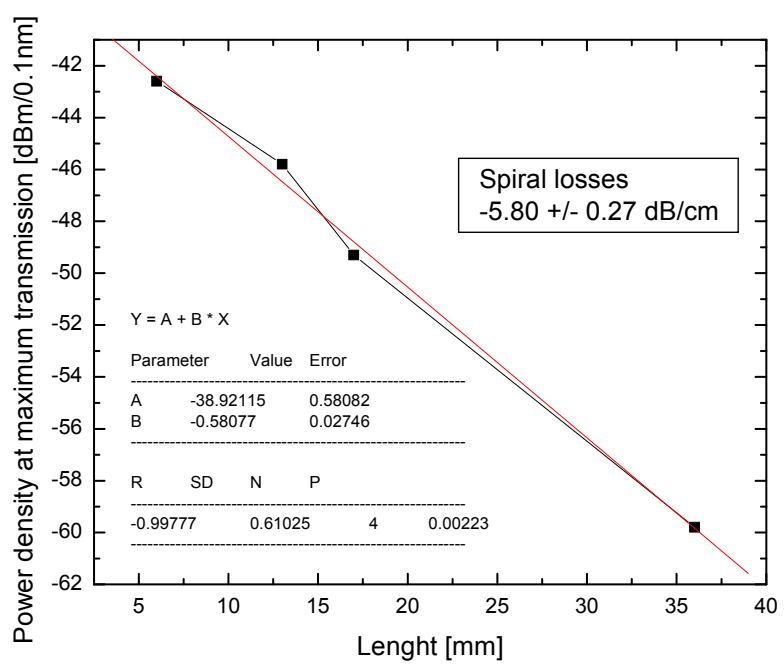

Fig. 14. Propagation losses for a set of four different spirals.

\subsection{Sensing the Biodiesel blends.}

Each photonic wire waveguide was tested with different kinds of Biodiesel/Diesel blends. The different fuels were added manually on top of the sensing box. Using equation 1, we can predict the behavior of the spiral with the fuel on top; this model uses the measured transmission for pure Biodiesel and pure Diesel shown in Fig. 1 and the confinement factor of each structure (20\% for regular waveguides).

Each measurement sequence consists of two parts. First, we measure the transmission spectrum of the waveguide configuration without fuel on top of the sensing area. Secondly, we measure the transmission after we added a fuel mixture on top of the sensing area. Finally, the transmission is normalized by subtracting both measurements. This way, the normalized spectrum already takes into account the waveguide propagation loss.

Fig. 15 plots the theoretical and the measured transmission of a $1.1 \mathrm{~cm}$ spiral. Here the differences in transmission between wires with standard Diesel and with Biodiesel are in the order of $2 \mathrm{~dB}$ for the $1665 \mathrm{~nm}$ region. The oscillating behavior is attributed to the Fabry-Pérot behavior found also in the spiral characterization. 
We carried out a second experiment with the $1.1 \mathrm{~cm}$ long spiral, to confirm our results (Fig. 16), but in this case using a Diesel/Biodiesel blend in the sensing box. As expected, the difference between pure Diesel and the blend decreases but is still clearly visible.

Note that for some wavelengths, the normalized transmission has values above 0dB. This would mean that when fuel (an absorbing medium) is present on the spiral, the transmission increases as compared to the situation without the presence of fuel. We attribute this is to a decrease in the scattering losses ${ }^{(13)}$. We believe the lower index contrast obtained from (Biodiesel-SOI) in comparison with, (Air-SOI), reduces the propagation losses..

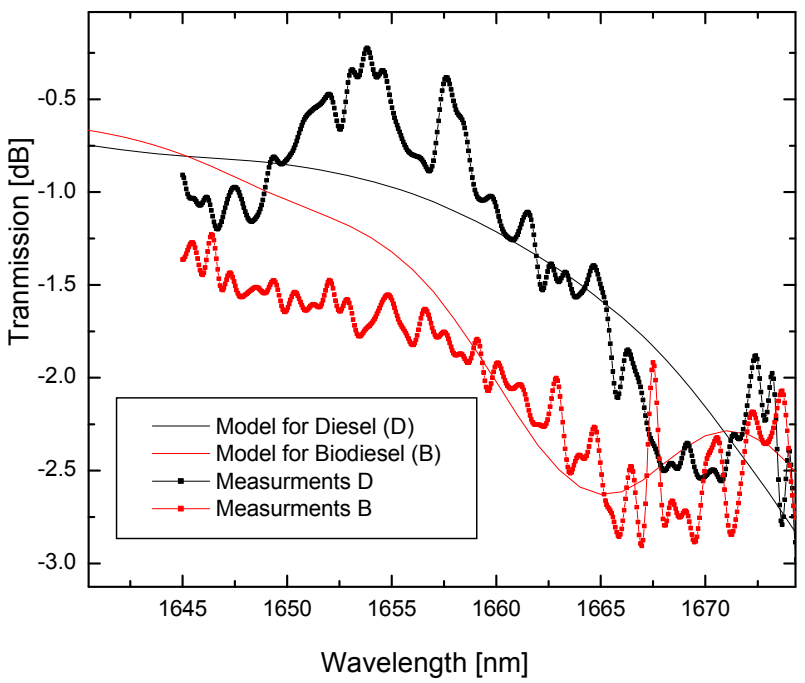

Fig. 15. Measurement and model comparison for the Biodiesel Diesel transmission spectrum for a $1.1 \mathrm{~cm}$ long spiral

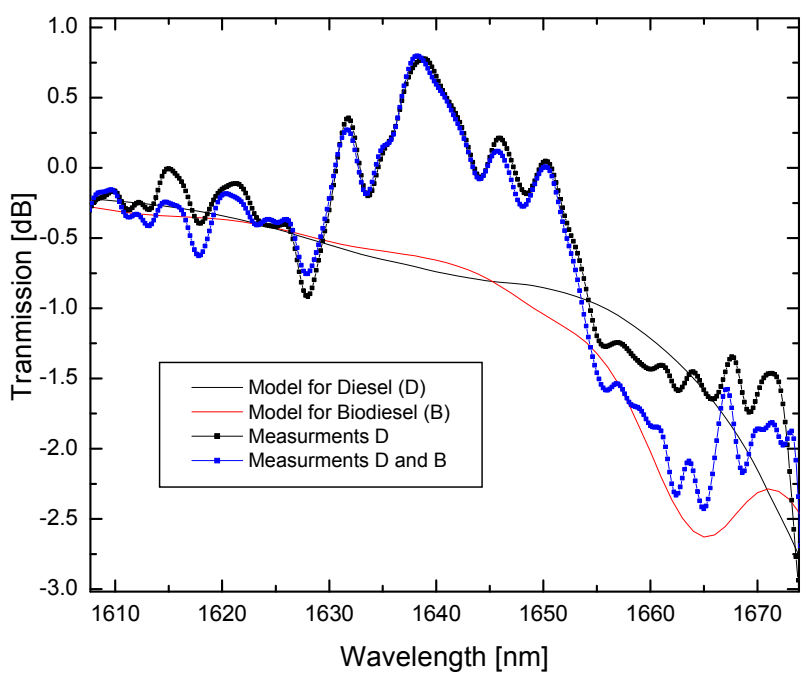

Fig. 16. Measurement and model comparison for the Diesel Blended Diesel transmission spectrum for a $1.1 \mathrm{~cm}$ long spiral.

Finally a $3.6 \mathrm{~cm}$ spiral was analyzed and is presented in Fig. 17. Again, the measurements agree with our expectations and the differences in absorption are around $4 \mathrm{~dB}$ (at $1665 \mathrm{~nm}$ ), making possible the identification of the fuel composition.

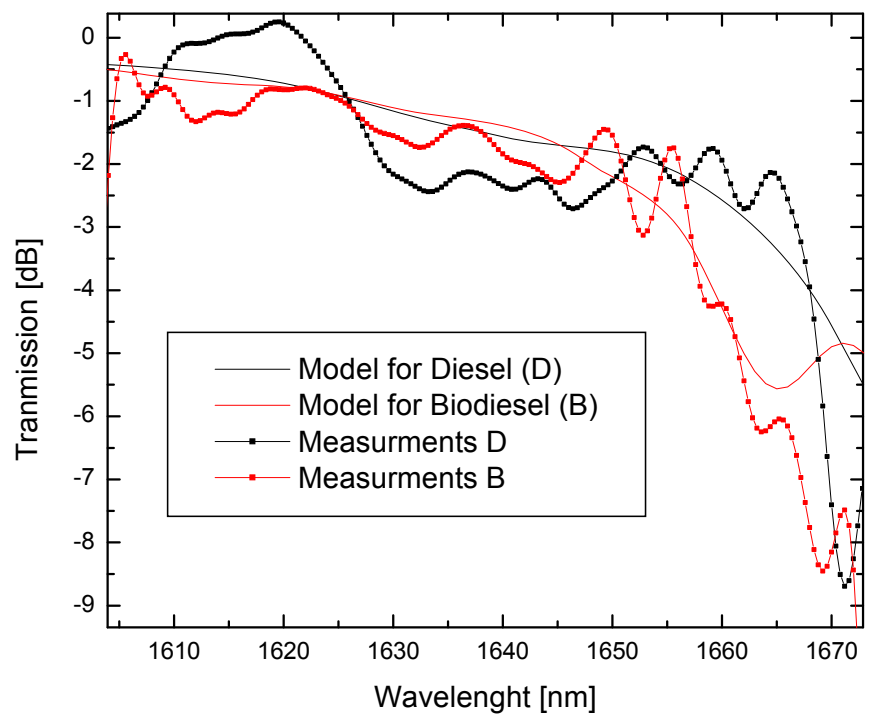

Fig. 17. Transmission spectrum measurement, and model comparison for a $3.6 \mathrm{~cm}$ spiral plus Biodiesel and Diesel 


\section{CONCLUSION}

We have measured Biodiesel/Diesel blends using SOI based spiral shaped waveguides. We obtained transmission differences of $2 \mathrm{~dB}$ and $4 \mathrm{~dB}$ for standard Diesel compared to Biodiesel, for a $1.1 \mathrm{~cm}$ and $3.6 \mathrm{~cm}$ spiral length respectively. We also presented a PCG spectrometer design able to perform Biofuel on-chip measurements.

The photonic wire created as a Biofuel sensor works in agreement with our expectations and we found correspondence with the theoretical predicted values and the experimental ones.

\section{REFERENCES}

[1] N. D. Mortimer, P. Cormack, M. A. Elsayed and R. E. Horne., "Evaluation of the comparative energy, global warming and socio-economic cost and benefits of Biodiesel", Sheffield Resources research unit school of environment and development, (2003).

[2] Maria Fernanda Pimentela, Grece M.G.S. Ribeiro , Rosenira S. da Cruz, Luiz Stragevitch, José Geraldo. Pacheco Filho, Leonardo S.G. Teixeira. "Determination of Biodiesel content when blended with mineral Diesel fuel using infrared spectroscopy and multivariate calibration", Microchemical Journal, Vol. 82, 201-206( 2006)

[3] Knothe, Gerhard. "Rapid Monitoring of Transesterification and Assessing Biodiesel Fuel Quality by Near-Infrared Spectroscopy Using a Fiber-Optic Probe", JAOCS 76 (1999)

[4] Rosane Falate, Karen Nike and Pedro Ramos da Costa Neto, Eduardo Cação Jr., Marcia Muller, Hypolito José Kalinowski and José Luís Fabris, "Alternative technique for Biodiesel quality control using an optical fiber long period", Quim. Nova 30, pp. 1677-1680, (2007)

[5] Ling, Meng Yee. "Design and fabrication of a near-infrared spectroscopy system for sensing applications", Erasmus Mundus M.Sc.in Photonics Thesis,(2008)

[6] J. Brouckaert, G. Roelkens, S. Selvaraja, W. Bogaerts, P. Dumon, S. Verstuyft, Z. Yu, D. Van Thourhout, R. Baets. "Miniature Integrated Spectrometer Fabricated on a Silicon-on-Insulator Substrate", United States : Leos Annual Meeting, (2008)

[7] Joost Brouckaert, Wim Bogaerts, Pieter Dumon, Dries Van Thourhout, Roel Baets. "Planar Concave Grating Demultiplexer With Hight Reflective Bragg Reflector Facets", IEEE Photonics technology letters, 20,(2008)

${ }^{[8]}$ W. H. Wang, Y. Z. Tang, Y. X. Wang, H. C. Qu, Y. M. Wu, T. Li, J. Y. Yang, Y. L. Wang, and M. Liu. "Etcheddiffraction-grating-based planar waveguide demultiplexer on silicon-on-insulator", Opt. Quantum Electronics 36, $559-566(2004)$

[9] Q. Xu, V. R. Almeida, R. R. Panepucci, and M. Lipson. "Experimental demonstration of guiding and confining light in nanometer-size low-refractive-index material" Opt. Lett 29, 14, 1626-1628 , (2004)

[10] "CAMFR", http://camfr.sourceforge.net/index.html,(2008)

[11] "fimmwave", http://www.photond.com/products/fimmwave/fimmwave2.htm.

[12] Taillaert, Dirk. "Grating Couplers as Interface between Optical Fibres and Nanophotonic Waveguides", Doctoral Thesis. Belgium : Gent University, (2004).

[13] Arthur Nitkowski, Long Chen, Michal Lipson. "Cavity-enhanced on-chip absorption spectroscopy using microring resonators", OPTICS EXPRESS 16, 16, 11930-11936.(2008) 